\title{
Competencia y eficiencia bancaria en el Perú mediante el indice de Boone ${ }^{\mathrm{I}}$
}

\section{Competition and bank efficiency in Peru through the Boone index}

\author{
William Miguel Jiménez Rivera ${ }^{2}$ \\ Universidad Privada del Norte \\ william.jimenez@upn.pe \\ https://orcid.org/0000-0002-7940-8409
}

Recibido: 15/08/2018 - Aceptado: 27/05/2020 - Publicado: 18/08/2020

\section{RESUMEN}

En el presente trabajo se estudia el nivel de competencia y eficiencia bancaria en el Perú para el periodo 2011 - 2016 con datos de frecuencia mensual, a través de la estimación por la técnica de datos de panel del índice de Boone para el sistema crediticio bancario en global y desagregado en cuatro tipos de créditos, como son: mayoristas, minoristas, de consumo e hipotecarios. Dada la importancia de un sistema bancario eficiente y competitivo para el desarrollo de los países este estudio muestra evidencia de la evolución del Índice de Boone, uno de los indicadores más usado por la nueva organización industrial empírica para medir la competencia y eficiencia en las industrias. En concreto se deja evidencia sobre la hipótesis de que la concentración aumenta la competencia y con ello la eficiencia, no muestra resultados del todo claros.

Palabras clave: Competencia bancaria; Función de costos translogarítmica; Índice de Boone.

JEL: G21; L11

(C) Los autores. Este artículo es publicado por Pensamiento Crítico de la Facultad de Ciencias Económicas, Universidad Nacional Mayor de San Marcos. Este es un artículo de acceso abierto, distribuido bajo los términos de la licencia Creative Commons Atribucion - No Comercia_Compartir Igual 4.0 Internacional. (http://creativecommons.org/licenses/by-nc-sa/4.0/) que permite el uso no comercial, distribución y reproducción en cualquier medio, siempre que la obra original sea debidamente citada. 


\section{ABSTRAC}

In the present work the level of competition and banking efficiency in Peru is studied for the period 20112016 with monthly frequency data, through the estimation by the panel data technique of the Boone index for the banking credit system in global and disaggregated into four types of loans, such as wholesalers, retailers, consumer and mortgage. Given the importance of an efficient and competitive banking system for the development of countries, this study shows evidence of the evolution of the Boone Index, one of the indicators most used by the new empirical industrial organization to measure competition and efficiency in industries. Specifically, evidence is left on the hypothesis that concentration increases competition and thus efficiency, does not show clear results at all.

Keywords: Bank competition; translogarithmic cost function; Boone Index.

JEL: G21; L11. 


\section{Introducción}

La magnitud del sistema bancario dentro del sistema financiero nacional es importante, según el Informe de evolución del sistema financiero 2016 de la Superintendencia de Banca y Seguros (SBS), al 31 de diciembre de $2016^{3}$, los bancos representan el $84 \%$ de total del sistema indirecto. Además, el sistema bancario peruano se caracteriza por tener elevados niveles de concentración a nivel de activos, créditos y patrimonio, durante el periodo que abarca este estudio sus niveles de concentración para los cuatro bancos más grandes superan en 75\%; los niveles de rentabilidad de los activos (ROA) alcanzan niveles del 18 al 20\% ${ }^{4}$, considerados altos, a decir, incluso de la Superintendencia de Banca, Seguros y AFPs del Perú, (SBS 2016, p. 17) "la rentabilidad de la banca múltiple permanece elevada". Sin embargo, los niveles de cartera pesada permanecen bajos, alrededor del $2 \%$ en promedio.

Para ver una comparación internacional en cuanto al margen financiero, es decir, el diferencial de tasas activas y pasivas, en la figura 1 podemos apreciar que el diferencial de tasas ha tenido un comportamiento decreciente en el tiempo. Así el Perú muestra un promedio de casi 20\% para los años 2000-2005 y 2006-2010, en el 2011 y 2012 está por encima de $16 \%$ y para los años siguientes llega a niveles menores al 15\%. Niveles por debajo de los mostrados por economías como Brasil y Paraguay, pero muy por encima de los que registra el promedio de América Latina y el Caribe (ALC) de alrededor de 6\%, el promedio de los países de ingreso mediano (clasificación otorgada también a Perú) que es ligeramente superior al promedio de países de ALC y un poco más lejos de los niveles que se muestran para el mundo donde el nivel promedio de diferencial de tasas bordea llega a niveles menores al 6\% anual. Visto en el periodo 2000 al 2016, los spreads de la banca peruana están, en promedio, 6 puntos porcentuales por encima de spreads en países de ingresos medios y unos puntos adicionales si lo vemos respecto al promedio mundial.

Según Mauricio (2016, p. 34) el sistema bancario peruano, al registrar uno de los más altos márgenes o spread del mundo, puede ser considerado como uno de los países con los peores spreads del mundo". 


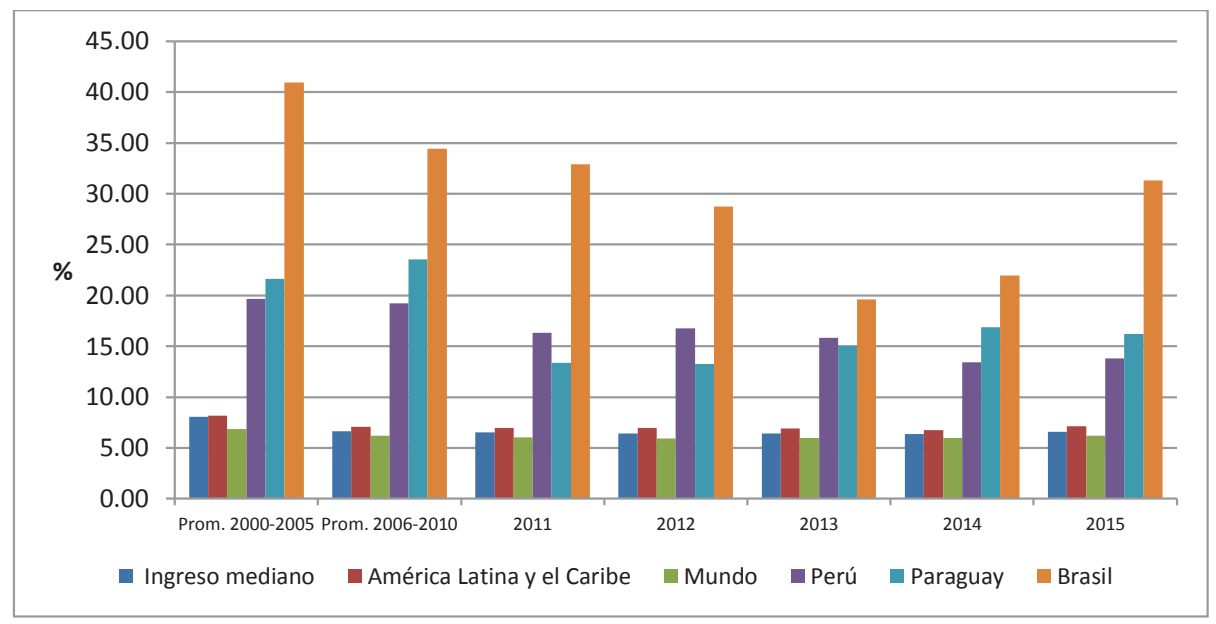

Figura 1. Diferencial de tasas de interés: tasa activa menos pasiva, \% (Países y bloques seleccionados 2000-2015)

Fuente: Banco Mundial. Elaboración: Propia

En este contexto, mediante el índice de Boone, este trabajo se propone analizar la evolución del grado de competencia de la banca peruana con las herramientas académicas planteadas por la economía de organización industrial y aplicadas en el caso concreto del sistema bancario. En particular se busca medir el grado de competencia a nivel de segmentos de colocaciones ${ }^{5}$ y probar la hipótesis de eficiencia con datos mensuales en el período 2011-2016.

Uno de los temas más controversiales en el ámbito económico es el relacionado al diagnóstico y cuantificación del poder de mercado, en particular, la cuestión que relaciona los niveles de competencia con el poder de mercado de la industria bancaria y con los niveles de eficiencia del mismo, por tener un papel clave en la intermediación de recursos financieros. Sin embargo, la forma de brindar evidencia al respecto no hay acuerdo entre los especialistas, en lo que sigue de este apartado se señala por qué se justifica la metodología aquí utilizada.

La competencia en el sector financiero es importante por varias razones. Al igual que en otras industrias, el grado de competencia en el sector financiero puede afectar la eficiencia de la producción de servicios 
financieros. (Claessens y Laeven, 2004), (Zuleta y otros, 2016). Además, los sistemas bancarios menos competitivos pueden ser más costosos y exhibir una menor calidad de servicios, lo que proporciona menos financiamiento y alentando menos crecimiento. (van Rixtel, 2008), (Claessens y Laeven, 2004), (Jopen, 2013).

Por lo anterior, promover una mayor competencia se hace necesaria puesto que la mayor competencia entre las entidades de crédito puede influir favorablemente en el bienestar de los hogares y las empresas. Van Rixtel (2008), citando a Bikker (2004) señala que también es favorable para la evolución macroeconómica general, en el sentido que las políticas de traspaso (de la tasa de interés) de la política monetaria se hacen más efectivas en sistemas bancarios más competitivos y eficientes.

En muchos países en desarrollo como en el Perú, la búsqueda de mayor competencia y eficiencia del sistema se ha promovido a través de la entrada de bancos extranjeros. A través del proceso de privatización de bancos nacionales privados o privados quebrados, se buscó que la banca extranjera traiga más recursos y a la vez una mayor eficiencia, también una presión más competitiva sobre los bancos nacionales sector bancario. (Beck, 2006).

Sin embargo, este proceso de ingreso de banca extranjera y los procesos de crisis también han acentuado los niveles de concentración bancaria (Zurita, 2014). En Perú, la aplicación del denominado Programa de Consolidación del Sistema Financiero, y el cambio del marco regulatorio generó como efecto colateral, una importante reducción del número de oferentes del sistema bancario, y por ende un importante incremento de los indicadores de concentración bancaria. (Jopen, 2013). Para evaluar las implicaciones de estos cambios es necesario medir el impacto de la consolidación en la industria bancaria sobre la estructura del mercado y el comportamiento de las entidades (Zurita, 2014).

Demirgüç-Kunt y otros. (2003) investigan el impacto del banco concentración y normativa sobre eficiencia bancaria. Encuentran que la concentración bancaria tiene un efecto negativo y significativo en la eficiencia del sistema bancario, excepto en países ricos con sistemas financieros bien desarrollados y más libertades económicas. Por su parte, Martín (2009) señala que la concentración bancaria puede desembocar 
en ciertos comportamientos de mercado que pueden no ser del beneficio común y que incluso pueden tener efectos perniciosos para el resto del mercado, generando una concentración no solo de mercado, sino también de riesgos.

Los estudios sobre el nivel de competencia se dividen en dos grupos fundamentales: enfoques estructurales y enfoques no estructurales. (Zurita, 2014). Los primeros son enfoques que recogen indicadores de la estructura del mercado como la concentración para evaluar impactos en el mercado (Las hipótesis de Estructura Conducta Resultado y la de eficiencia son representativas). Por otra parte, el enfoque alternativo rechaza que los indicadores estructurales proporcionen un buen indicador del comportamiento del mercado, por tanto, para conocer las conductas y estrategias de las empresas, así como las características de la competencia, la nueva organización industrial empírica (NOEI) propone indicadores no estructurales a partir de la observación directa de la conducta de las empresas en el mercado. (Zuleta, 2016). Estos modelos no consideran la concentración del mercado en sus análisis. (Zurita, 2014).

El enfoque no estructural desarrolla los modelos de Iwata, Bresnahan-Lau y Panzar-Rosse. (Zurita, 2014). Respecto de estos nuevos enfoques muchos trabajaos empíricos se han desarrollado, encontrando diversos resultados. Claessens y Laeven (2004) aplicaron la metodología Panzar-Rosse a los sistemas bancarios de 50 países para analizar las condiciones competitivas de los sistemas bancarios, encontrando que, además de la participación de bancos extranjeros, las medidas de la estructura del sistema no proporcionaron ninguna indicación de la competitividad de los mercados, al menos no como esperaban.

Ante la necesidad de brindar evidencia sobre la competencia y su relación con la concentración, y dado problema que en el análisis del sector bancario la competencia no puede medirse directamente, ya que no es posible disponer fácilmente de datos sobre costes y, con frecuencia, tampoco sobre los precios de los productos bancarios individuales, Boone propone la metodología del indicador que lleva su nombre, el mismo que medir el efecto de la eficiencia sobre los resultados en términos de beneficios o, como en el caso del presente documento, se utiliza como variante las cuotas del mercado. 


\section{Metodología y Data}

Como se indicó en el apartado anterior la medida desarrollada en el estudio es el indicador de Boone, este indicador se sustenta en las siguientes premisas, las empresas más eficientes aumentan su participación de mercado por lo que captan mayores utilidades. Luego, este efecto es mayor cuanto mayor sea la competencia en el mercado. Según Alarcón y Ormazábal (2015, p.7) un aspecto central para garantizar este indicador es que "en los mercados competitivos, las empresas que son más eficientes trasladarán al menos parte de sus ganancias de eficiencia a sus clientes en forma de reducciones de precios y, por consiguiente, obtendrán mayores cuotas de mercado"

\section{Derivación del modelo de Boone susceptible de estimación empírica.}

Aunque Boone ha desarrollado modelos teóricos para diferentes tipos de mercados en distintos trabajos entre el 2000 y el 2008, aquí se toma como referencia la aplicación de Leuvensteijn y otros (2007), los cuales se basaron en Boone y otros (2004) para su aplicación de la teoría a la industria bancaria, siendo considerado el primer trabajo en aplicar este modelo a la industria bancaria.

Para la aplicación del modelo en bancos, partimos que, en una industria bancaria donde cada banco $i$ ofrecediferentes tipos de productos denominados $q_{i}$ y enfrenta una curva de demanda como la de la ecuación 1:

$$
p\left(q_{i}, q_{j} \neq i\right)=a-b q_{i}-d \sum_{j \neq i} q_{j}
$$

con un costo marginal constante $m c_{i}$, $a$ es un intercepto que puede interpretarse como el precio que un cliente está dispuesto a pagar a cualquier banco que ofrezca la primera unidad de producto, $b$ mide la sensibilidad al precio de un cliente con respecto al producto ofrecido por el banco $i$, mientras que $d$ es la sensibilidad del precio de un cliente al mismo producto ofrecido por los bancos rivales $q_{j \neq i}$. Así, $d$ representa el grado de sustitución entre los productos. 
Dado que el banco tiene un costo marginal constante, maximiza su utilidad $\pi_{i}=\left(p_{i}-m c_{i}\right) q_{i}$; escogiendo el óptimo nivel de producción $q_{i}$ que resuelve el siguiente problema:

$$
\left\{\left(a-2 b q_{i}-d \sum_{j i}^{\operatorname{Max}} q_{j}-m c_{i}\right) q_{i}\right\}
$$

El modelo exige además que asumamos que $a>m c_{i}$ (de lo contrario, el beneficio es negativo desde el principio) y $0<d \leq b$ (lo que significa que los productos no pueden ser sustitutos perfectos), la condición de primer orden (CPO)de un equilibrio Nash-Cournot para la firma i, establece que:

$$
a-2 b q_{i}-d \sum_{j \neq i} q_{j}-m c_{i}=0
$$

En una industria con $\mathrm{N}$ bancos con niveles de productos positivos, sin pérdida de generalidad, la solución simultánea de las CPO nos lleva a:

$$
q_{i}\left(m c_{i}\right)=\frac{\left(2 \frac{b}{d}-1\right) a-\left(2 \frac{b}{d}+N-1\right) m c_{i}+\sum_{j} m c_{j}}{(2 b+d(N-1))\left(2 \frac{b}{d}-1\right)}
$$

Esta ecuación relaciona el nivel de producción y los costos marginales.

Boone señala que la competencia se puede mejorar a través de dos canales: a través de un aumento en $d$ (los productos se vuelven sustitutos más cercanos) y mediante una disminución de los costos de entrada. Boone et al, 2004 demostraron que, efectivamente, la competencia redistribuía el producto (en términos monetarios) de las empresas ineficientes a las más eficaces. Si los bancos obtienen beneficios $\pi$ sin considerar los costos de entrada $\epsilon$, se entiende que,la empresa bancaria ingresa a competir siempre que $\pi_{>} \epsilon$ en el equilibrio. Por tanto, de $\pi_{i}=\left(p_{i}-m c_{i}\right) q_{i} \mathrm{y}$ de la ecuación anterior, se concluye que los beneficios dependen del costo marginal de manera cuadrática como la presentada a continuación:

$$
\pi_{i}=b\left[q\left(m c_{i}\right)\right]^{2}
$$


Por lo tanto, del análisis anterior se puede inferir que un mayor nivel de competencia aumenta los beneficios relativos de una empresa en relación con una empresa menos eficiente. Finalmente, el cambio de producción hacia las empresas más eficientes significa que, en un entorno más competitivo, las empresas más eficientes también ganarán una cuota de mercado. Por lo tanto, la competencia también conducirá a que las empresas más eficientes obtengan una mayor porción del mercado. Los autores consideran el logaritmo de los beneficios de cada banco en función de una variable proxy del costo marginal (el cual es inobservable) dada por la razón de costos variables $c_{i}$ entre ingresos $p_{i}$. La función de beneficios queda de la siguiente manera.

$$
\ln \pi_{i}=\alpha+\beta \frac{c_{i}}{p_{i}}+u_{i}
$$

Al tener la variable dependiente en logaritmos, esta ecuación es equivalente a estimar la relación utilizando algún nivel de referencia arbitrario para la función de beneficios, que será capturado en la constante $\alpha$. En tanto que, la medida de competencia en términos de beneficios relativos será recogida por el coeficiente $\beta$, que representa una media de la elasticidad ingreso, el cual será negativo puesto que los bancos menos eficientes tendrían menores beneficios relativos. La expresión $u_{i}$ es el ya conocido término de error.

Leuvensteijn y otros (2007); Leuvensteijn ((2008) aplican el modelo Boone (2004) para la industria bancaria, para lo cual introdujeron importantes cambios para hacerlo más funcional. En primer lugar, calculan el costo marginal mediante la estimación de una función multiproducto translogarítmica que se alinea más con la teoría. En segundo lugar, emplean el nivel de participación de mercado de cada firma en lugar de los beneficios dado que también es consistente con la hipótesis de eficiencia. Murillo (2011, p. 55-56), menciona tres ventajas importantes a partir de este cambio.

i. el modelo teórico descrito está en concordancia con el impacto de la eficiencia sobre las cuotas de mercados y su relación con la competencia (consistente con la hipótesis de eficiencia). 
ii. las participaciones de mercado son siempre positivas, mientras que el rango de beneficios incluye valores negativos (o pérdidas).

iii. utilizar las participaciones de mercado logra identificar cada tipo de negocio bancario (créditos, depósitos, etc.), mientras que los márgenes de beneficios por lo general no se encuentran diferenciados por cada línea de negocio.

Por lo tanto, Leuvensteijn y otros (2007), Leuvensteijn (2008) aplican el modelo Boone utilizando el enfoque de participación de mercado y estiman el indicador de Boone de acuerdo con la siguiente ecuación (suponiendo que haya $\mathrm{N}$ bancos en el sistema),

$$
\ln S_{i}=\alpha+\beta \ln m c_{i}+u_{i}
$$

Donde

- $\quad$ In $S_{i}$ es el logaritmo neperiano del nivel de participación de cada banco $i$, para el producto q; $S_{i}=\frac{q_{i}}{\sum_{J=1}^{N} q_{j}}$

- $\quad \alpha$ es la constante

- $\quad \beta$ es el indicador de Boone para la industria bancaria en un segmento específico

- $\quad$ In $m c_{i}$ es el logaritmo neperiano del costo marginal del banco $i$.

En primera instancia, se debe encontrar una mayor participación de mercado en aquellos bancos con menores costos marginales, es decir, esperaríamos que el coeficiente $\beta$ sea negativo. Además, conforme haya más competencia en la industria más fuerte debería ser esta relación y más grande, sería el valor de $\beta$ (en términos absolutos). Según Alarcón y Ormazabal (2015 p. 15) "el indicador de competencia de Boone es precisamente el valor de este parámetro $\beta$ : valor absoluto mayor del parámetro es indicativo de mayor competencia"

Además, de la ecuación 7 se desprende que, bajo competencia perfecta, la participación de mercado de cada banco es tan insignificante que se aproximaría a cero, por lo que la variable dependiente tiende al infinito negativo. Y dado que cada agente es tomador de precios, y el precio es igual al costo marginal, el término que acompaña al coeficiente $\beta$ es 
constante, lo que implica que tiende a menos infinito. Murillo (2011 p. 67) remarca que si "por el contrario, $\alpha=\beta=0$ es consistente con un escenario monopólico, donde la participación del único banco es $S_{i}=1$ ”

\section{La Función de Costos}

Toda función de costos mide el costo mínimo al cual el empresario obtiene un volumen dado de producción, a partir de la combinación de diferentes factores productivos, y dados los precios de mercado. Sobre el tipo de actividad que ejercen las entidades crediticias, no se ha llegado a un análisis concluyente a pesar de la amplia literatura teórica y empírica que tratan el sector financiero. No obstante, en la literatura económica se menciona dos enfoques para la medición contable de la actividad bancaria, estos son: por un lado, el enfoque de la producción y de otro el de la intermediación.

El enfoque de la producción, se basa en la función de producción clásica, donde los bancos utilizan los factores de capital y trabajo para obtener un output conformado por el conjunto de depósitos y préstamos. Este output se mide en unidades físicas, es decir, por el número de transacciones realizadas. En tanto que los costos de producción solo se limitan a los costos operativos. De acuerdo con Caminero y Mancebo (2016) a este enfoque se le critica no tomar en cuenta los costos por intereses, los mismo que se constituyen en gran proporción de los costos totales de la industria bancaria.

Por otra parte, el enfoque de intermediación considera que los bancos son intermediarios de servicios financieros en forma de créditos. Los montos monetarios de los préstamos e inversiones son las cuentas contables definidas como output que se genera a partir de insumos como el capital o infraestructura y el trabajo utilizados, además, los costos operativos más los costos por fondeo se constituyen como los costos relevantes, en tanto que para los depósitos tiene como opción ser considerados como insumos o productos. Este enfoque es preferido sobre el de producción, dada la ventaja que representa la facilidad de encontrar datos relevantes, y es el usado en esta investigación.

La forma de la función de costos transmite información sobre la tecnología usada por cada empresa. Dada la trascendencia de los costos para el estudio de la eficiencia y la competitividad de las industrias se busca 
el método y las funciones que presenten el mejor ajuste de estimación. Xavier (2013, p. 8) indica que "hasta la década de los 70's las funciones Cobb-Douglas y la de costos de elasticidad constante (CES) eran las normalmente usadas. Posteriormente se comienza a buscar nuevas alternativas, debido a una serie de limitaciones que presentan"Por ejemplo, sobre las especificaciones Cobb-Douglas y CES se señala que no permiten explicar las economías de escala ni el nivel de complementariedad entre los insumos, además de restricciones técnicas, como las elasticidades de sustitución unitaria en el caso de la Cobb-Douglas y constante entre cada par de insumos en la funcion CES.

Dadas las dificultades de las funciones de costos antes mencionadas surge en la literatura económica el uso las funciones de costos flexibles, como la translogarítmica estándar y la translogarítmica generalizada, entre otras. Croce, Macedo, y Triunfo (2000, p. 10) citando a Diiewrt señalan, "Diewert define una función flexible como aquélla que tiene suficientes grados de libertad para permitir una aproximación de segundo orden a una función de costos arbitraria dos veces diferenciable y linealmente homogénea"

La función de costos translogarítmica es un método paramétrico para representar a través de la expansión de Taylor de segundo orden la función de costos a través de un punto cualquiera. Sin embargo, no está excenta de crítica,los autores señalan hasta cuatro especificaciones de la funcion translogarítimica. Al respecto Croce, Macedo, y Triunfo, (2000, p. 10) señalan que "pese a ser la más utilizada, no es capaz de medir el comportamiento de los costos cuando no hay producción, es decir, no es útil para medir economias de alcance globales"

\section{Estimación de la Función de Costos Translogarítmica (FCT)}

A partir de la ecuación (7) tenemos dos variables que debemos considerar al estimar el indicador de Boone: la cuota de mercado $S_{i}$ y el costo marginal $m c_{\mathrm{i}}$. Los datos sobre los productos ofrecidos por los bancos (tales como colocaciones, depósitos, etc.) en una industria bancaria están comúnmente disponibles pues los declaran periódicamente en sus estados financieros. El cálculo del costo marginal resulta ser mucho más complicado.

La estimación de la función de costo marginal, se obtiene de una función de costo translogarítmica, de acuerdo a la literatura revisada. En este 
trabajo seguiremos la metodología de estimación de Roengpitya (2010) que es, a la vez, una variante de la estimación propuesta por Leuvensteijn y otros (2007).

Como ya se indicó, la función de costos translogarítmica es una expansión de segundo orden de la serie de Taylor en unidades físicas de outputs y en precios de los inputs con variables en las formas logarítmicas naturales. Así, la FCT a estimar en este trabajo es:

$$
\begin{aligned}
\ln C_{i t}^{h}=\alpha_{0}+ & \sum_{h=1}^{H-1} \alpha_{h} d_{i}^{h}+\sum_{t=1}^{T-1} \delta_{t} d_{t}+\sum_{h=1}^{H} \sum_{j=1}^{K} \beta_{j h} \ln x_{i j t} d_{i}^{h} \\
& +\sum_{h=1}^{H} \sum_{j=1}^{K} \sum_{k=1}^{K} \gamma_{j k h} \ln x_{i j t} \ln x_{i k t} d_{i}^{h}+v_{i t}
\end{aligned}
$$

Donde:

- $\quad$ n $c_{i t}^{h}$ es el logaritmo natural de la función de costo de cada banco $i$ en el periodo $t$

El subíndice " $h$ " se refiere a cada tipo de banco

- $\quad d_{i}^{h}$ es una categórica del tipo de banco

- $\quad d_{t}$ es una categórica de tiempo

- $\quad x_{i k t}$ corresponde a distintas variables de control (activos, tasas, etc.)

- $\quad v_{i t}$ es el término de perturbación

A partir de la ecuación (8) obtenemos el costo marginal de cada banco para cada uno de los segmentos de productos de interés:

$$
m c_{i 1 t}^{h}=\frac{c_{i t}^{h}}{x_{i 1 t}}\left(\beta_{1 h}+2 \gamma_{1 l h} \ln x_{i l t}+\sum_{k=1, \ldots, K ; k \neq 1} \gamma_{1 k h} \ln x_{i k t}\right) d_{i}^{h}
$$

Donde, el subíndice "1" corresponde al tipo de producto y así sucesivamente. Por ejemplo, 1: créditos mayoristas; 2 : créditos minoristas; etc. 
Las variables explicativas $\mathrm{X}_{\mathrm{ijt}}$ se pueden agrupar en dos categorías: componentes de salida: productos y componentes de entrada: insumos. Aquí se considera cuatro productos: créditos mayoristas, créditos minoristas, créditos de consumo y créditos hipotecarios. Los precios de tres insumos incluyen tasas salariales, tasas de financiamiento y tasa de capital físico. Los productos ingresan a la ecuación como una cantidad en unidades monetarias, mientras que los precios de los insumos ingresan a la ecuación como una tasa.

Sin pérdida de generalidad, defino $\mathrm{j}=1, \ldots, K y k=1, \ldots, K$ de la siguiente manera.

En la cual la clasificación para $k$ y $j$ es la siguiente:

$j=k=1 \quad$ para precio de la mano de obra (variable "pers")

$j=k=2 \quad$ para precio de los fondos prestables (variable "fond")

$j=k=3 \quad$ para precio del capital físico (variable " $k f i s^{\prime \prime}$ )

$j=k=4 \quad$ para créditos mayoristas (variable "mayor")

$j=k=5$ para créditos minoristas (variable "minor")

$j=k=6 \quad$ para créditos de consumo (variable "cons")

$j=k=7 \quad$ para créditos hipotecarios (variable "hipot")

Además, se imponen restricciones adicionales a los coeficientes de la FCT

$$
\begin{aligned}
& \beta_{1}+\beta_{2}+\beta_{3}=1 \\
& r_{1, k}+r_{2, k}+r_{3, k}=0, \mathrm{k}=1,2,3 \\
& r_{k, 1}+r_{k, 2}+r_{k, 3}=0 \quad k=4,5,6 \\
& r_{j, k}=r_{k, j} \forall_{j, k}=1, \ldots \ldots, K
\end{aligned}
$$

La primera y la segunda condición indican la suposición de la homogeneidad lineal en los precios de los insumos, lo que significa que las tres elasticidades del precio de los insumos $\left(\beta_{i}, \mathrm{i}=1,2,3\right)$ suman 1 , mientras que los términos al cuadrado y cruzados de todas las variables explicativas $\left(y_{i, j}\right.$ 
) suman cero. La última condición en la ecuación (13) es la restricción de simetría tal que $r_{j, k}=r_{k, j}$ para $j, k=1 \ldots K$.

Luego de desarrollar la función de costos translogarítmica, la función a estimar es la siguiente:

$$
\begin{aligned}
& \operatorname{LnCT}=\alpha_{0}+\delta_{t} d_{t}+\operatorname{Ln}(k f i s)+\beta_{1}[\operatorname{Ln}(\text { pers })-\operatorname{Ln}(k f i s)] \\
& +\beta_{2}[\operatorname{Ln}(\text { fond })-\ln (k f i s)]+\beta_{4} \operatorname{Ln}(\text { mayor })+\beta_{5} \operatorname{Ln}(\text { minor }) \\
& +\beta_{6} \operatorname{Ln}(\text { cons })+\beta_{7} \operatorname{Ln}(\text { hipot })+r_{11}[\operatorname{Ln}(\text { pers })-\operatorname{Ln}(k f i s)]^{2} \\
& +2 r_{12}[\operatorname{Ln}(\text { pers })-\operatorname{Ln}(k f i s)][\operatorname{Ln}(\text { fond })-\operatorname{Ln}(k f i s)] \\
& +2 r_{14}[\operatorname{Ln}(\text { mayor })][\operatorname{Ln}(\text { pers })-\operatorname{Ln}(k f i s)] \\
& +2 r_{15}[\operatorname{Ln}(\text { minor })][\operatorname{Ln}(\text { pers })-\operatorname{Ln}(k f i s)] \\
& +2 r_{16}[\operatorname{Ln}(\text { cons })][\operatorname{Ln}(\text { pers })-\operatorname{Ln}(k f i s)] \\
& +2 r_{17}[\operatorname{Ln}(\text { hipot })][\operatorname{Ln}(\text { pers })-\operatorname{Ln}(k f i s)] \\
& +r_{22}[\operatorname{Ln}(\text { fond })-\operatorname{Ln}(k f i s)]^{2} \\
& +2 r_{24}[\operatorname{Ln}(\text { mayor })][\operatorname{Ln}(\text { fond })-\operatorname{Ln}(k f i s)] \\
& +2 r_{25}[\operatorname{Ln}(\text { minor })][\operatorname{Ln}(\text { fond })-\operatorname{Ln}(k f i s)] \\
& +2 r_{26}[\operatorname{Ln}(\text { cons })][\operatorname{Ln}(\text { fond })-\operatorname{Ln}(k f i s)] \\
& +2 r_{27}[\operatorname{Ln}(\text { hipot })][\operatorname{Ln}(\text { fond })-\operatorname{Ln}(k f i s)]+r_{44} L n^{2} \text { (mayor) } \\
& +2 r_{45} \operatorname{Ln} \text { (mayor) } \operatorname{Ln}(\text { minor })+2 r_{46} \operatorname{Ln} \text { (mayor) } \operatorname{Ln}(\text { cons }) \\
& +2 r_{47} \operatorname{Ln} \text { (mayor) } \operatorname{Ln} \text { (hipot) }+r_{55} L n^{2} \text { (minor) } \\
& +2 r_{56} \operatorname{Ln} \text { (minor) } \operatorname{Ln} \text { (cons) }+2 r_{57} \operatorname{Ln} \text { (minor) } \operatorname{Ln} \text { (hipot) } \\
& +r_{66} \operatorname{Ln}^{2} \text { (cons) }+2 r_{67} \operatorname{Ln}(\text { cons }) \operatorname{Ln}(\text { hipot })+r_{77} \operatorname{Ln}^{2} \text { (hipot) }
\end{aligned}
$$

Finalmente, usando la definición general de los costos marginales se obtienen el costo marginal de cada tipo de crédito. Así, por ejemplo, los costos marginales para los préstamos mayoristas (mayor) se define como:

$$
C M g_{i 4 t}=\frac{\partial C M g_{i 4 t}}{\partial x_{i 4 t}}=\frac{\partial \operatorname{Ln}\left(C M g_{i 4 t}\right)}{\partial \operatorname{Ln}\left(x_{i 4 t}\right)}
$$

Es decir, para poder determinar $\frac{\partial \operatorname{Ln}\left(C M g_{i 4 t}\right)}{\partial \operatorname{Ln}\left(x_{i 4 t}\right)}=\frac{\partial \operatorname{Ln}(C T)}{\partial \operatorname{Ln}(\text { mayor })}$ se debe diferenciar la ecuación (14) con respecto al Ln(mayor) y así tenemos:

$$
\begin{gathered}
\frac{\partial \operatorname{Ln}(C T)}{\partial \operatorname{Ln}(\text { mayor })}=\beta_{4}+2 r_{44} \operatorname{Ln}\left(x_{i 4 t}\right)+\sum_{k \neq 4} r_{4 k} \operatorname{Ln}\left(x_{i 4 t}\right)+\sum_{j \neq 4} r_{j 4} \operatorname{Ln}\left(x_{i j t}\right) \\
\frac{\partial \operatorname{Ln}(C T)}{\partial \operatorname{Ln}(\text { mayor })}=\beta_{4}+2 r_{44} \operatorname{Ln}\left(x_{i 4 t}\right)+2 \sum_{k \neq 4} r_{4 k} \operatorname{Ln}\left(x_{i k t}\right)
\end{gathered}
$$




$$
\begin{aligned}
\frac{\partial \operatorname{Ln}(C T)}{\partial \operatorname{Ln}(\text { mayor })} & =\beta_{4}+2 r_{14} \operatorname{Ln}(\text { pers })+2 r_{24} \operatorname{Ln}(\text { fond })+2 r_{34} \operatorname{Ln}(\text { kfis }) \\
& +2 r_{44} \operatorname{Ln}(\text { mayor })+2 r_{45} \operatorname{Ln}(\text { minor })+2 r_{46} \operatorname{Ln}(\text { cons }) \\
& +2 r_{47} \operatorname{Ln}(\text { hipot })
\end{aligned}
$$

Entonces, el costo marginal para el crédito mayorista es:

$$
\begin{aligned}
C M g_{i 4 t}= & \frac{\partial C T}{\partial x_{i 4 t}}=\frac{C T}{x_{i 4 t}}\left[\beta_{4}+2 r_{44} \operatorname{Ln}\left(x_{i 4 t}\right)+2 \sum_{k \neq 4} r_{4 k} \operatorname{Ln}\left(x_{i k t}\right)\right] \\
C M g_{(\text {mayor })}= & \frac{C T}{\text { mayor }}\left[\beta_{4}+2 r_{14} \operatorname{Ln}(\text { pers })+2 r_{24} \operatorname{Ln}(\text { fond })+2 r_{34} \operatorname{Ln}(\text { kfis })\right. \\
& +2 r_{44} \operatorname{Ln}(\text { mayor })+2 r_{45} \operatorname{Ln}(\text { minor })+2 r_{46} \operatorname{Ln}(\text { cons }) \\
& \left.+2 r_{47} \operatorname{Ln}(\text { hipot })\right]
\end{aligned}
$$

La tabla 1 describe cada una de las variables utilizadas en la estimación de la función de costos translogarítmica y la obtención del costo

\begin{tabular}{|c|c|c|}
\hline Variable & Nombre & Definición \\
\hline CT & Costo total & Costo operativo más gasto financiero en miles de soles \\
\hline \multicolumn{3}{|c|}{ Productos } \\
\hline Mayor & Créditos mayoristas & Créditos corporativos y a grandes empresas en miles de soles \\
\hline Minor & Créditos minoristas & Créditos a medianas y microempresas en miles de soles \\
\hline Cons & Créditos de consumo & Créditos a consumo y tarjetas de crédito en miles de soles \\
\hline Hipot & Créditos hipotecarios & Créditos para vivienda en miles de soles \\
\hline \multicolumn{3}{|l|}{ Insumos } \\
\hline Pers & Precio de la mano de obra & Gastos en personal divididos por el total de empleados \\
\hline Fond & Precio de los fondos prestables & Gastos por intereses entre el total de captaciones \\
\hline Kfis & Precio del capital físico & Depreciación del periodo sobre el nivel de activo fijo. \\
\hline \multicolumn{3}{|c|}{ Variables de Control } \\
\hline Moros & Tasa de morosidad & $\begin{array}{l}\text { Cociente entre el importe de los créditos morosos y el total de } \\
\text { préstamos concedidos }\end{array}$ \\
\hline Tend & Tendencia & Medida para ver el efecto de la tecnología en el tiempo \\
\hline
\end{tabular}
marginal de cada producto crediticio en este estudio. Los datos del costo total y de los créditos han sido deflactadas por el índice de precios al consumidor, IPC vigente (base 2008).

Tabla 1

Definición de variables e indicadores a emplearse en las estimaciones de la Función de Costos Translogarítmica e Indicador de Boone

Elaboración propia 
Los datos a utilizarse provienen de las hojas de balance y de estados de resultados que los bancos presentan mensualmente a la Superintendencia de Banca, Seguros y AFPs y la Superintendencia del Mercado de Valores. La variable Índice de precios se obtiene de la página web del Banco central de Reserva. Todas las series se trabajan en periodicidad mensual y cubren desde el año 2011 al 2016.

Los bancos son agrupados en tres categorías:

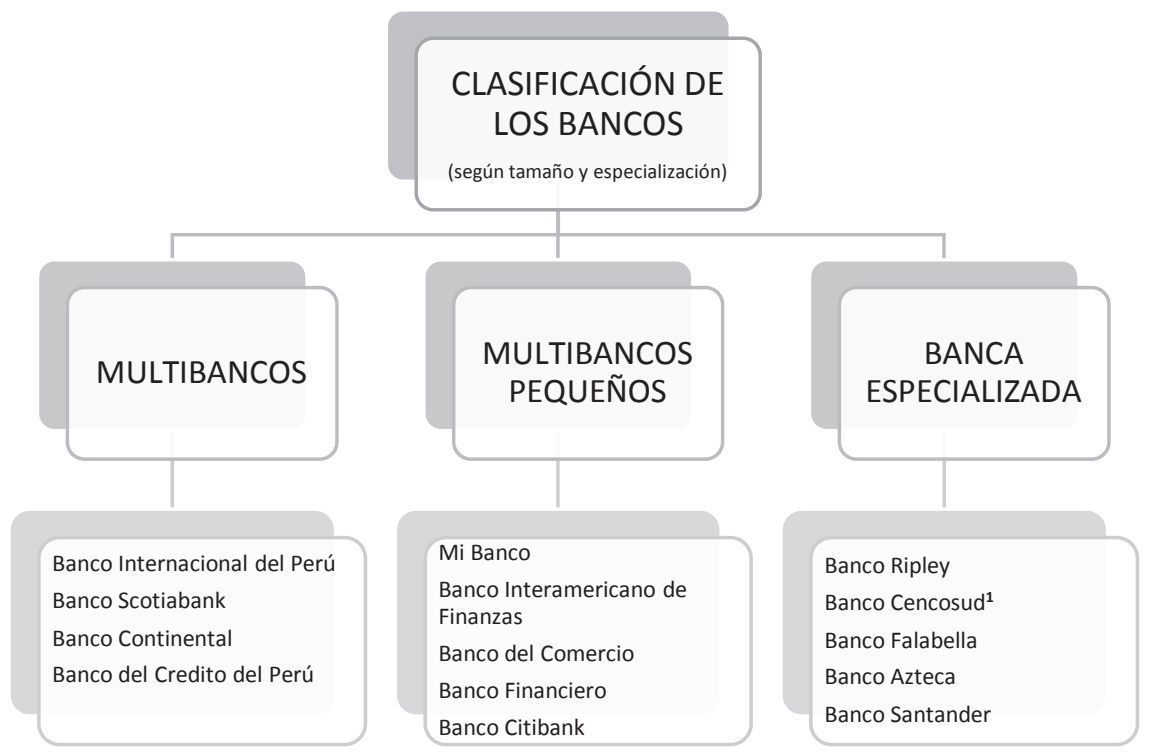

Figura 2. Clasificación de los bancos según su tamaño y especialización

Elaboración propia

Se denomina Multibancos grandes a los bancos líderes del mercado en cuanto a participación de mercado de activos, colocaciones y depósitos, según las estadísticas presentadas, entre estos 4 bancos concentran más del $80 \%$ del mercado, los Multibancos pequeños son aquellos que tienen presencia menor en los distintos segmentos de mercado que se ha divido los créditos para el estudio, finalmente son bancos especializados aquellos bancos que tienen una alta concentración de créditos en un segmento en particular. 
La tabla 2 presenta los promedios de las variables utilizadas, para el total del sistema y por tipo de banco, 2011.1 -2016.12 (los valores monetarios se expresan en soles de 2008). Podemos ver los siguientes resultados:

- Los bancos grandes tienen en promedio, poco más de 10 veces el valor de las colocaciones que los Multibancos pequeños y 30 veces el valor de las colocaciones que el valor de las colocaciones de la banca especializada.

- Los bancos grandes presentan, en promedio, menores costos en mano de obra, fondeo y de capital físico que el promedio del mercado. Además, presentan una menor tasa de morosidad.

- La banca especializada está tiene, en promedio más colocaciones que los Multibancos pequeños en los segmentos de créditos mayoristas y en los créditos de consumo.

Tabla 2

Promedios de las variables utilizadas, para el total del sistema y por tipo de banco, 2011.1 -2016.12 (los valores monetarios se expresan en soles de 2008)

\begin{tabular}{|l|l|l|l|l|}
\hline \multicolumn{1}{|c|}{ Variables } & \multicolumn{1}{|c|}{$\begin{array}{c}\text { Total Sistema } \\
\text { Bancario }\end{array}$} & \multicolumn{1}{|c|}{$\begin{array}{c}\text { Multibancos } \\
\text { Grandes }\end{array}$} & \multicolumn{1}{c|}{$\begin{array}{c}\text { Multibancos } \\
\text { pequeños }\end{array}$} & \multicolumn{1}{c|}{$\begin{array}{c}\text { Banca } \\
\text { especializada }\end{array}$} \\
\hline Costos Totales (miles de S/) & $74.014,1$ & $189.434,9$ & $28.740,6$ & $22.184,6$ \\
\hline Créditos totales (miles de S/) & $10.781 .652,9$ & $31.267 .759,6$ & $3.066 .777,3$ & $1.057 .514,8$ \\
\hline Créditos mayoristas (miles de S/) & $8.492 .596,6$ & $18.969 .970,8$ & $1.482 .500,7$ & $1.633 .579,2$ \\
\hline Créditos minoristas (miles de S/) & $1.315 .748,6$ & $2.086 .450,8$ & $954.403,3$ & $3.206,9$ \\
\hline Créditos consumo (miles de S/) & $1.904 .567,1$ & $4.687 .089,2$ & $610.714,6$ & $828.803,8$ \\
\hline Créditos hipotecarios (miles deS/) & $2.448 .237,0$ & $5.524 .248,7$ & $358.954,6$ & $10.245,3$ \\
\hline Precio de la mano de obra (Soles) & 14,87 & 13,42 & 18,53 & 10,45 \\
\hline Costo de fondeo (\% mensual) & 0,38 & 0,25 & 0,34 & 0,58 \\
\hline Costo de capital físico (\%) & 1,63 & 1,07 & 1,22 & 2,91 \\
\hline Tasa de morosidad (\%) & 3,14 & 2,05 & 2,43 & 5,49 \\
\hline
\end{tabular}

Fuente: SBS. Elaboración propia

\section{Marco Teórico}

El tema de la competencia en el sector bancario ha despertado mucho interés en los últimos años debido a su papel crucial en la actividad no financiera. Y si bien, su medición y, sobre todo, sus efectos resultan controversiales, 
muchos documentos teóricos han intentado explicar las consecuencias de la competencia en diversos ámbitos como el acceso al crédito y su costo, la calidad de los servicios financieros, la estabilidad de los sistemas financieros y su efecto en el desarrollo económico. Para Freixas y Rochet (2008, p. 69)“una modelación correcta de estos temas es crucial para una mejor comprensión de algunos resultados empíricos o enigmas que aún carecen de una base teórica completa"

El hecho de que la competencia sea una noción compleja, y por lo tanto no directamente observable, ha resultado en el desarrollo de muchos métodos para su evaluación. La evaluación de la competencia en la industria bancaria tiene una extensa discusión. La literatura sobre la medición de la competencia generalmente se clasifica en dos principales de las corrientes. Con base en la Organización Industrial Tradicional, las primeras investigaciones se centraron en los vínculos estructura-desempeño del mercado, denominado el paradigma Estructura-Conducta-Resultados (SCP, P del inglés Performance) que establece, a priori, que la probabilidad de alcanzar mejores resultados aumenta con la concentración del mercado. Algunos autores, sin embargo, plantearon dudas sobre la fiabilidad del paradigma Estructura-Conducta-Resultado y las medidas estructurales asociadas de la competencia. En respuesta a las deficiencias encontradas en el enfoque estructural, se han desarrollado medidas no estructurales de competencia. El objetivo de las medidas de la Nueva Organización Industrial es evaluar directamente la conducta competitiva de las empresas.

Según el paradigma estructura-conducta-resultado, el entorno del mercado tiene un impacto directo a corto plazo en la estructura del mercado. La estructura del mercado se refiere al nivel de concentración del mismo, el cual impacta de forma directa en las decisiones de la empresa hacia una conducta de tipo competitiva o colusiva, lo que a su vez afecta su desempeño en el mercado. Ahí, los efectos de retroalimentación ocurren de tal forma que la performance del mercado puede influir en su comportamiento y su composición, o el comportamiento puede afectar la composición del mercado.

Un importante sector de investigadores a cuestionado los aspectos metodológicos y la aplicación empírica del modelo SCP y sus hipótesis mencionadas, proponiendo alternativas a este método, como el estadístico-H de 
Panzar y Rosse -que es una aproximación de la sensibilidad del ingreso a los cambios en el costo de los factores- y el mark-up (diferencial) del valor de los activos totales en relación a su costo marginal estimado (cuya equivalencia en términos porcentual es el índice de Lerner), además del Indicador Boone, el desarrollo de este último se presenta más adelante.

Las nuevas corrientes de economía de organización industrial aplicada plantean que, en principio es la empresa y no la industria la unidad básica de análisis y en cuanto a la metodología, se emplea con mayor énfasis el análisis longitudinal con series de tiempo en vez del análisis de corte transversal; Así Murillo (2011, p. 33) indica que "la investigación empírica debe centrarse en estimar los parámetros de conducta de la empresa y de la industria más que por estudiar la interacción entre la estructura y resultados"

Los nuevos modelos que se desarrollan se denominan modelos no estructurales, en el caso aplicado al mercado bancario, se basan en estudiar la forma en que los bancos responden ante cambios en los niveles de costos y su relación con la evolución de sus ingresos, ingresos financieros específicamente.

\section{El estado de la cuestión}

El estudio de la competencia y la eficiencia del sistema bancario han sido abordados con bastante amplitud por estudiosos de la región como del país, sin embargo, los estudios predominantes son, a nivel de Panzar y Rosse, los presentados por Cortez (2006), Cortez (2008) y por Céspedes y Orrego (2014), sin embargo, tienen como dificultad principal que presentan resultados generales para el sistema bancario, es decir, se calcula un indicador único para todo el sistema bancario. Del Pozo (2008) también aplica la metodología al sector de microfinanzas en el Perú y encuentra que el sector presenta un nivel de competencia monopolística.

El índice de Lerner también se ha aplicado para medir la competencia en la industria bancaria peruana. Por ejemplo, Jopen (2013) analiza el desempeño del Índice de Lerner — bajo el supuesto de una función de costos tipo translog, como una aproximación del poder de mercado, encuentra que los bancos con mayor cantidad de activos (denominados como bancos grandes) han mostrado una marcada tendencia creciente de sus indicadores de poder de mercado; mientras que en el caso de los bancos con 
menor cantidad de activos (denominados como bancos pequeños) sucede lo opuesto, con una menor tendencia. Por tanto, el tamaño de los bancos influye en los márgenes de tasas de interés. Por su parte Mayorca y Aguilar (2016) indican que el Índice de Lerner como indicador de competencia revela el poder que tiene cada institución para establecer un precio por encima de su costo marginal, es decir, revela su poder de mercado.

El Colombia el trabajo de Zuleta y otros (2014) en el marco teórico competencia realiza una investigación para el sistema bancario colombiano entre 1995y 2014, muestra los indicadores estructurales de concentración como HHI y estiman los índices no estructurales: el Índice de Lerner y el indicador de competencia $\mathrm{H}$, de Panzar y Rosse (P-R). Además del modelo de eficiencia no paramétrico de frontera estocástica. Encuentran que la concentración no influye claramente en los índices de rentabilidad, mediante Lerner encuentran que existe un bajo poder de mercado y mediante P-R concluyen que el sector financiero colombiano tiene un nivel de competencia intermedio que se ubica entre la competencia perfecta y el monopolio. Finalmente, señalan que, si bien la ineficiencia técnica ha venido reduciéndose, hay oportunidades de seguir mejorando.

Un enfoque novedoso para medir la relación entre competencia y concentración es presentado por Morón, Tejada y Villacorta (2010) quienes estiman un modelo para la demanda residual corregida por la calidad del producto, del que obtienen elasticidades de demanda que asumen como indicadores de niveles de competencia para el periodo 2001-2010. La particularidad de este estudio es que se enfoca en un nivel específico de productos financieros (básicamente distintos tipos de créditos y depósitos). Sin embargo, en opinión de Aguilar (2016, p. 21) "la principal dificultad del estudio es que no diferencia entre los diferentes segmentos de intermediación financiera ni el tamaño de los intermediarios".

A nivel internacional, Murillo (2011) estima el Indicador de Boone para evaluar el poder de mercado y el grado de competencia de la banca venezolana tanto en el mercado de créditos como el de depósitos. Encuentra que el mercado de créditos es más competitivo que el de depósitos. Una dificultad de este trabajo es que estudia de forma agrupada a los créditos y también a los depósitos, por ende, no distingue entre los tipos que hay al interior de cada uno de esos mercados. Alarcón y Ormazabal 
(2013), hacen un estudio para la banca chilena mediante el indicador de competencia de Boone. Encuentran que la concentración y competencia bancaria están directamente relacionadas, y resaltan la existencia de niveles de competencia diferentes entre distintos tipos de créditos.

En relación al Índice de Boone para Perú, Aguilar (2016) analiza la competencia en el mercado microcréditos vinculándola con la eficiencia de las empresas y su participación en el mercado entre el 2003 - 2009. Distingue entre créditos a MYPES y "otros". Encuentra que la competencia se ha intensificado en el primero de ellos, aún la mayor concentración observada. Finalmente, Aguilar (2016, p. 4) señala que "en el mercado de créditos "otros" la competencia se ha intensificado entre cajas municipales y rurales más no así, entre bancos y financieras".

En relación a los trabajos anteriores, acá se mostrará el índice de Boone para el mercado bancario del Perú, tomando segmentos de créditos específicos en la consideración que no todos los bancos compiten en todos los segmentos dada su tamaño, capacidad operativa, estrategias de crecimiento, entre otros factores.

\section{Resultados y Discusión}

El cálculo del Índice de Boone requiere estimar los costos marginales de los créditos y obtener la participación de mercado de los bancos. En primera instancia, se trabajó el sistema como un todo, considerando el total de créditos como crédito único. Posteriormente, se estudia los créditos por segmento de acuerdo al criterio definido por el Banco central de Reserva.

La tabla 3 presenta los resultados de las estimaciones de la Función de costos translogarítmica para el sistema bancario como un todo y para los tres segmentos del sistema determinados para el presente estudio. La metodología es mediante la regresión de un panel mediante mínimos cuadrados generalizados factibles, ponderado por la tasa de morosidad bancaria.De la tabla 6 podemos apreciar que, en términos generales, las funciones de costos estimadas presentan buenos niveles de ajuste, alrededor del 99\%. La mayoría de los coeficientes estimados son estadísticamente significativos al 1\%. Principalmente los que son usados para el cálculo del costo marginales, los cuales se han resaltado en negrita. 
Pensamiento Crítico Vol. 25. N¹

Tabla 3

Estimación de Función de Costos Translogarítmica para el Crédito total de la banca peruana, por tipo de bancos. 2011-2016

\begin{tabular}{|c|c|c|c|c|c|}
\hline \multicolumn{6}{|c|}{ Variable dependiente: $\operatorname{Ln}(\mathrm{CT})$-Ln(kfis) } \\
\hline Explicación de variables & Coeficientes & Total Sistema & Multibancos & $\begin{array}{l}\text { Multibancos } \\
\text { pequeños }\end{array}$ & $\begin{array}{c}\text { Banca } \\
\text { especializada }\end{array}$ \\
\hline \multirow{2}{*}{ Ln pers - Ln kfis } & \multirow{2}{*}{$\beta_{1}$} & $0.723^{* * *}$ & $1,472^{* * *}$ & $2,325^{* * *}$ & $-0,255$ \\
\hline & & 6,50 & 3,11 & 5,15 & $-0,99$ \\
\hline \multirow{2}{*}{ Ln fond - Ln kfis } & \multirow{2}{*}{$\beta_{2}$} & 0.062 & $-1,787^{* * *}$ & 0.656 & $1,282^{* * *}$ \\
\hline & & 0,59 & $-3,12$ & 1,32 & 5,34 \\
\hline \multirow{2}{*}{ Lncred } & \multirow{2}{*}{$\beta_{4}$} & $0.654^{* * *}$ & $-0,201$ & $5,516^{* * *}$ & $1,063^{* * *}$ \\
\hline & & 16,17 & $-0,43$ & 3,44 & 1,22 \\
\hline \multirow{2}{*}{ Ln moros } & \multirow{2}{*}{$\beta_{5}$} & $0.035^{* * *}$ & $0,087^{* * *}$ & $0.346^{* * *}$ & 0.001 \\
\hline & & 3,52 & 2,96 & 9,93 & 0,04 \\
\hline \multirow{2}{*}{ Tend } & \multirow{2}{*}{$\beta_{6}$} & $0.001^{* *}$ & $0,003^{* * *}$ & -0.000 & $0.004^{* * *}$ \\
\hline & & 2,34 & 4,46 & $-0,89$ & 5,63 \\
\hline \multirow{2}{*}{ (Ln pers -Ln kfis) 2} & \multirow{2}{*}{$\gamma_{11}$} & $0.045^{* * *}$ & 0,019 & $0.071^{* * *}$ & -0.015 \\
\hline & & 4,81 & 0,94 & 6,61 & $-0,72$ \\
\hline \multirow{2}{*}{$\begin{array}{l}\text { (Ln pers - Ln kfis)(Ln fond } \\
\text { - Ln kfis) }\end{array}$} & \multirow{2}{*}{$2 \gamma_{12}$} & $-0.017 * * *$ & $-0,061^{* * *}$ & $-0.052 * * *$ & $0.046^{* * *}$ \\
\hline & & $-2,75$ & $-2,73$ & $-7,19$ & 3,58 \\
\hline \multirow{2}{*}{ (Ln cred)(Ln pers -Ln kfis) } & \multirow{2}{*}{$2 \gamma_{14}$} & $-0.017 * * *$ & $-0,036^{* * *}$ & $-0.083^{* * *}$ & $0.039 * * *$ \\
\hline & & $-4,34$ & $-2,64$ & $-6,02$ & 3,94 \\
\hline \multirow{2}{*}{ (Ln fond-Ln kfis)2 } & \multirow{2}{*}{$\gamma_{22}$} & $0.025^{* * *}$ & $0,089^{* * *}$ & $0.068^{* * *}$ & $-0.084^{* * *}$ \\
\hline & & 3,03 & 4,45 & 8,43 & $-3,99$ \\
\hline \multirow[b]{2}{*}{ (Ln cred)(Ln fond - Lnkfis) } & \multirow[b]{2}{*}{$2 \gamma_{24}$} & $0.016^{* * *}$ & $0,081^{* * *}$ & 0.012 & $-0.054^{* * *}$ \\
\hline & & 4,70 & 5,04 & $\begin{array}{l}\text { 0,73 (Flores \& } \\
\text { Watts, 2012) }\end{array}$ & $-5,92$ \\
\hline \multirow{2}{*}{ Lncred ${ }^{\wedge} 2$} & \multirow{2}{*}{$\gamma_{44}$} & $0.007^{* * *}$ & $0,030^{* * *}$ & $-0.147^{* * *}$ & $-0.029 * * *$ \\
\hline & & 3,35 & 2,04 & $-2,80$ & $-5,31$ \\
\hline \multirow{2}{*}{ Ln $\operatorname{moros}^{\wedge} 2$} & \multirow{2}{*}{$\gamma_{55}$} & \begin{tabular}{|l|}
$-0.008^{*}$ \\
\end{tabular} & $-0,021$ & $-0.110^{* * *}$ & $0.022^{* * *}$ \\
\hline & & $-1,85$ & $-0,90$ & $-7,42$ & 4,19 \\
\hline \multirow{2}{*}{ tend $^{\wedge} 2$} & \multirow{2}{*}{$\gamma_{66}$} & $-0,000^{* * *}$ & $-0,000^{* * *}$ & $-0,000^{* * *}$ & $-0,000^{* * *}$ \\
\hline & & $-8,55$ & $-5,70$ & $-3,14$ & $-4,05$ \\
\hline \multirow{2}{*}{ Intercepto } & \multirow{2}{*}{$\alpha$} & $-1.405^{* * *}$ & $6,667^{*}$ & $-3.876^{* * *}$ & $-1,525^{* * *}$ \\
\hline & & $-7,82$ & 1,74 & $-3,16$ & $-6,57$ \\
\hline Nro. De Bancos & & 14 & 4 & 5 & 5 \\
\hline Nro. Observaciones & & 1008 & 288 & 360 & 340 \\
\hline$R^{\wedge} 2$ Ajustado & & 0,998 & 0,997 & 0,991 & 0,994 \\
\hline & & $F(13,962)$ & $\mathrm{F}(3,271)$ & $\mathrm{F}(4,342)$ & $F(4,323)$ \\
\hline F lest de Efectos fijos & & 559,61 & 960.62 & 80,36 & 257,21 \\
\hline
\end{tabular}

Fuente: Estimaciones propias

Elaboración propia. El nivel de significancia viene indicado así. 1\%, ${ }^{* * *} ; 5 \%, * * ; 1 \%, *$. Los valores de errores estándar robustos aparecen debajo del coeficiente estimado. Los coeficientes en negrita han sido usados para el cálculo del costo marginal. 
La figura 3 representa el promedio de los costos marginales por cada banco para el periodo que va desde enero del 2011 a diciembre del 2016.

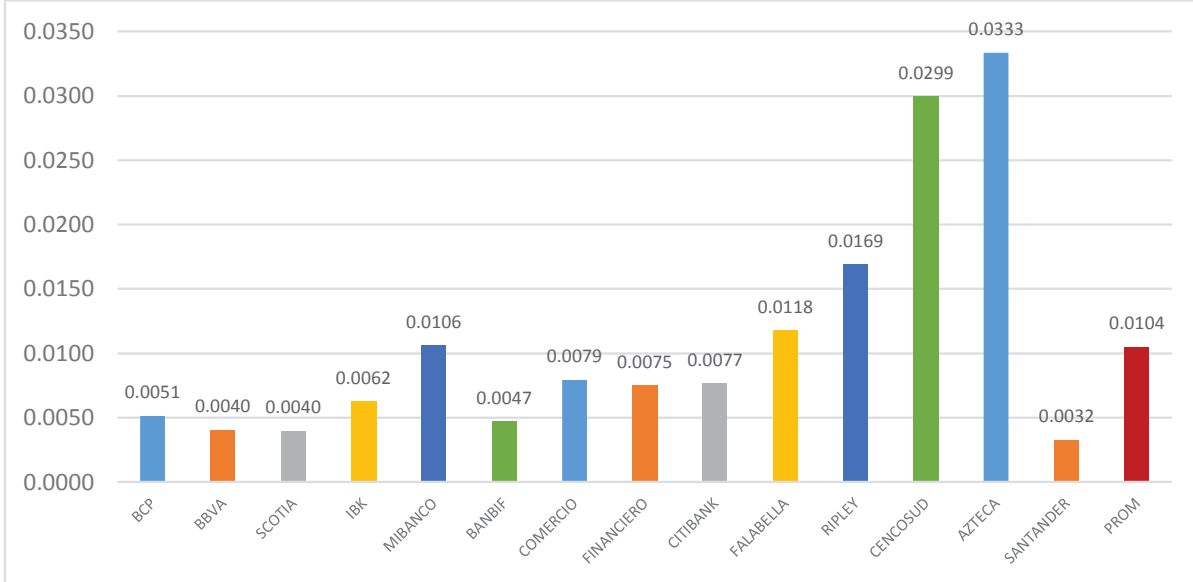

Figura 3. Promedio del costo marginal de los créditos bancarios 2011-2016,

(Unidades por cada sol)

Fuente: Cálculos en base a estimaciones propias. Elaboración propia

Se observa que, el promedio del costo marginal del sistema es de 0,0104 que equivaldría a 10.4 soles por cada mil soles de créditos colocados. Si vemos el desempeño individual de los bancos, el mejor promedio de costos marginales le corresponde al banco Santander con 0.0032, es decir, 3.2 soles por cada mil colocados, en segundo lugar, le siguen los bancos Scotiabank y el Banco Continental (BBVA) con costos de 4 soles por cada mil, lo cual no deja de sorprender puesto que son bancos más pequeños en activos, depósitos y colocaciones que el Banco de Crédito, pero con la coincidencia que pertenecen a la banca internacional. Más atrás viene el Banco Interamericano de Finanzas, el Banco de Crédito y el Banco Internacional (Interbank).

En cuanto a los bancos que presentan el peor desempeño promedio son el Banco Azteca, Cencosud y Ripley, los cuales para este estudio han sido clasificados como banca especializada por atender a un solo sector crediticio principalmente. El Banco Azteca tiene un promedio de 0,033 que representa 33 soles de costo marginal por cada 1000 soles colocados, el 
Banco Cencosud alcanza un costo marginal de 0,0299 y el banco Ripley un nivel de 0.0169. Los bancos Mibanco y Falabella presentan costos promedio similares al promedio del total del sistema. El estudio de la evolución de los costos marginales nos muestra mejores luces sobre el desempeño de los bancos en el periodo.

La estimación de la función de costos translogarítmica que se muestra en la tabla 4.

Tabla 4

Estimación de Función de Costos Translogarítmica por tipo de crédito de la banca peruana, $2011.1-2016.12$

\begin{tabular}{|c|c|c|c|c|}
\hline \multicolumn{2}{|c|}{ Variable dependiente: $\operatorname{Ln}(\mathrm{CT})$ - Ln(kfis) } & \multirow[b]{2}{*}{ Coef. estimado } & \multirow[b]{2}{*}{ t-Statistic } & \multirow[b]{2}{*}{ Prob. } \\
\hline Explicación de variables & Coeficiente & & & \\
\hline Ln pers - Ln kfis & $\beta_{1}$ & 0,038 & 0,48 & 0,63 \\
\hline Ln fond - Ln kfis & $\beta_{2}$ & 0,845 & 11,73 & 0,00 \\
\hline Ln mayor & $\beta_{4}$ & $-0,554$ & $-5,79$ & 0,00 \\
\hline Ln minor & $\beta_{5}$ & 0,167 & 4,34 & 0,00 \\
\hline Ln cons & $\beta_{6}$ & 0,891 & 2,12 & 0,00 \\
\hline Ln hipot & $\beta_{7}$ & $-0,526$ & $-9,34$ & 0,00 \\
\hline (Ln pers -Ln kfis)2 & $r_{11}$ & 0,061 & 5,74 & 0,00 \\
\hline $2^{*}($ Ln pers - Ln kfis) (Ln fond - Ln kfis) & $2 r_{12}$ & $-0,099$ & $-14,56$ & 0,00 \\
\hline $2^{*}($ Ln mayor)(Ln pers)-Ln kfis) & $2 r_{14}$ & $-0,010$ & $-3,33$ & 0,00 \\
\hline $2^{*}($ Ln menor)(Ln pers)-Ln kfis) & $2 r_{15}$ & $-0,004$ & $-1,61$ & 0,11 \\
\hline $2^{*}($ Ln cons)(Ln pers)-Ln kfis) & $2 r_{16}$ & 0,001 & 0,57 & 0,57 \\
\hline $2^{*}($ Ln hipot)(Ln pers)-Ln kfis) & $2 r_{17}$ & 0,009 & 3,91 & 0,00 \\
\hline (Lnfond-Ln kfis) 2 & $r_{22}$ & 0,101 & 13,09 & 0,00 \\
\hline $2^{*}($ Ln mayor) (Ln fond)-Ln kfis) & $2 r_{24}$ & 0,022 & 8,96 & 0,00 \\
\hline $2^{*}($ Ln menor)(Ln fond)-Ln kfis) & $2 r_{25}$ & 0,001 & 0,27 & 0,78 \\
\hline $2^{*}(\operatorname{Ln}$ cons $\left.)(\operatorname{Ln} f o n d)-\operatorname{Ln} k f i s\right)$ & $2 r_{26}$ & $-0,002$ & $-0,81$ & 0,42 \\
\hline $2^{*}($ Ln hipot)(Ln fond)-Ln kfis) & $2 r_{27}$ & $-0,010$ & $-4,57$ & 0,00 \\
\hline Ln mayor ${ }^{\wedge} 2$ & $r_{44}$ & 0,052 & 12,70 & 0,00 \\
\hline $2^{*}($ Ln mayor $)($ Ln minor $)$ & $2 r_{45}$ & $-0,006$ & $-3,04$ & 0,00 \\
\hline $2^{*}($ Ln mayor $)($ Ln cons $)$ & $2 r_{46}$ & $-0,020$ & $-18,22$ & 0,00 \\
\hline $2^{*}($ Ln mayor $)($ Ln hipot $)$ & $2 r_{47}$ & $-0,004$ & $-4,76$ & 0,00 \\
\hline Ln minor^2 & $r_{55}$ & 0,002 & 1,48 & 0,14 \\
\hline $2^{*}$ (Ln minor) (Ln cons) & $2 r_{56}$ & 0,000 & $-0,45$ & 0,65 \\
\hline $2^{*}($ Ln minor $)($ Ln hipot $)$ & $2 r_{57}$ & 0,001 & 1,72 & 0,09 \\
\hline
\end{tabular}


Tabla 4: Continuación...

\begin{tabular}{|c|c|c|c|c|}
\hline \multicolumn{2}{|c|}{ Variable dependiente: $\operatorname{Ln}(\mathrm{CT})$ - Ln(kfis) } & \multirow[b]{2}{*}{ Coef. estimado } & \multirow[b]{2}{*}{ t-Statistic } & \multirow[b]{2}{*}{ Prob. } \\
\hline Explicación de variables & Coeficiente & & & \\
\hline $\operatorname{Lncons}^{\wedge} 2$ & $r_{66}$ & $-0,017$ & $-5,97$ & 0,00 \\
\hline $2 *($ Ln cons)(Ln hipot) & $2 r_{67}$ & 0,013 & 7,60 & 0,00 \\
\hline Ln hipot^2 & $r_{77}$ & 0,010 & 4,59 & 0,00 \\
\hline Ln moros & $z_{1}$ & $-0,001$ & $-0,17$ & 0,87 \\
\hline Ln $\operatorname{moros}^{\wedge} 2$ & $z_{2}$ & $-0,012$ & $-2,83$ & 0,00 \\
\hline Tend & $z_{3}$ & 0,004 & 10,00 & 0,00 \\
\hline Ln tend ${ }^{\wedge} 2$ & $Z_{4}$ & 0,000 & $-8,46$ & 0,00 \\
\hline Intercepto & $\alpha$ & 6,211 & 11,22 & 0,00 \\
\hline Nro. Observaciones & & 989,000 & & \\
\hline$F(13,944)$ & & 796,750 & & \\
\hline $\mathrm{R}^{\wedge} 2$ Ajustado & & 0,999 & & \\
\hline
\end{tabular}

Fuente: Estimaciones propias. Elaboración propia.

La estimación se realizó mediante mínimos cuadrados ponderados factibles (FGLS) ponderada por el nivel de morosidad de cada banco. Incluye además el componente tendencial para medir si hubo efectos de los cambios tecnológicos en los costos bancarios, esta variable resulta estadísticamente significativa al $1 \%$ de significancia. El modelo en conjunto presenta un alto grado de ajuste, $99 \%$.

\section{Indicador de Boone por Segmentos crediticios}

Para la obtención del índice de Boone por segmentos se ha considerado a los bancos según su participación en los diferentes tipos de créditos, así, la tabla siguiente muestra qué bancos han sido considerados para el estudio de cada tipo de crédito.

Para calcular el Indicador de Boone, a menudo se ejecutan mediante la introducción de efectos fijos con el fin de tener en cuenta la heterogeneidad no observada. Según Florian (2014 p. 30) "trabajos recientes también controlan la endogeneidad al correr un enfoque instrumental". En este apartado presento el análisis por el método de momentos generalizado (GMM) siguiendo el procedimiento utilizado por Leuvensteijn y otros (2007) y Roengpitya (2010). 
Pensamiento Crítico Vol. 25. Nº1

Tabla 5

Clasificación de los Bancos según su participación en los diferentes segmentos crediticios

\begin{tabular}{|l|l|l|l|}
\hline \multicolumn{1}{|c|}{ Mayoristas } & \multicolumn{1}{|c|}{ Minoristas } & \multicolumn{1}{c|}{ Consumo } & \multicolumn{1}{c|}{ Hipotecario } \\
\hline \hline De Crédito & De Crédito & De Crédito & De Crédito \\
Scotiabank & Continental & Continental & Continental \\
Interbank & Scotiabank & Scotiabank & Scotiabank \\
Mibanco* & Interbank & Interbank & Interbank \\
Banbif & Mibanco & Mibanco & Mibanco \\
Comercio* & Banbif & Banbif & Banbif \\
Santander & Financiero & Comercio & Comercio* \\
Financiero & Comercio & Santander & Santander \\
Citibank & Citibank & Financiero & Citibank \\
& Santander* & Citibank & Ripley \\
& & Azteka & \\
& & Ripley & Cencosud \\
\end{tabular}

Elaboración propia a partir de la clasificación y estudio de la participación de los bancos en los diferentes tipos de créditos ${ }^{6}$.

Los resultados de la estimación del Índice de Boone para el sistema bancario peruano para el periodo 2011 al 2016 se presentan en la tabla adjunta.

Tabla 6

Estimación del Índice de Boone para la banca peruana por tipo de Crédito, Periodo, 20112016

\begin{tabular}{|l|l|l|l|l|l|}
\hline Tipo de Crédito & Indicador de Boone & \multicolumn{1}{|c|}{ t-statist } & \multicolumn{1}{|c|}{ Test J } & \multicolumn{1}{|c|}{ R2 } & Nro. de bancos \\
\hline Total & $-0,665^{* * *}$ & $-9,728$ & 0,00 & 0,997 & 14 \\
\hline Mayoristas & $0,026^{* *}$ & 1,936 & 0,00 & 0,997 & 8 \\
\hline Minoristas & $-0,785^{* * *}$ & $-22,546$ & 0,00 & 0,9930 & 9 \\
\hline Consumo & $-1,109^{* * *}$ & $-45,745$ & 0,00 & 0,998 & 14 \\
\hline Hipotecario & $-0,200^{* * *}$ & $-8,549$ & 0,00 & 0,992 & 8 \\
\hline
\end{tabular}

Elaboración a partir de Estimaciones propias. El nivel de significancia viene indicado así. 1\%, ${ }^{* * *} ; 5 \%,{ }^{* *} ; 1 \%$, *.

Se aprecia que, al estimar un solo parámetro de competencia, de acuerdo al indicador de Boone, la cartera de créditos de consumo presenta mayor competencia, siendo esta cartera en la que participan todos 
los bancos considerados en este estudio. Le sigue en nivel de competencia la cartera de créditos minoristas, segmento en el cual, además de los bancos es posible encontrar otras ofertas crediticias, principalmente de las denominadas empresas microfinancieras (IFIS). Los segmentos que presentan menores niveles de competencia y con resultados por debajo del promedio del sistema son, el segmento hipotecario, el cual es un crédito más selectivo y finalmente el segmento de créditos mayoristas en el que son pocos bancos los que compiten por atender a las grandes empresas y las corporaciones, este resultado podría resultar paradójico si consideramos que las empresas corporativas pueden acceder a financiamiento del mercado de valores lo que haría este segmento más competitivo.

En Sudamérica, Alarcón y Ormazábal (2013, p. 12) encuentran para el sistema bancario un Índice de Boone de -1,0498 para el periodo 20082009. En cuanto a los segmentos encuentran más competitiva la cartera de vivienda, seguida a la de consumo.

Si observamos la evolución del Índice de Boone para el mercado agregado, figura 4, es decir, créditos totales, puede observarse que la competencia en el sistema bancario se habría incrementado entre 2011 y 2015, pero en 2016 sufre un retroceso en sus niveles de competencia.

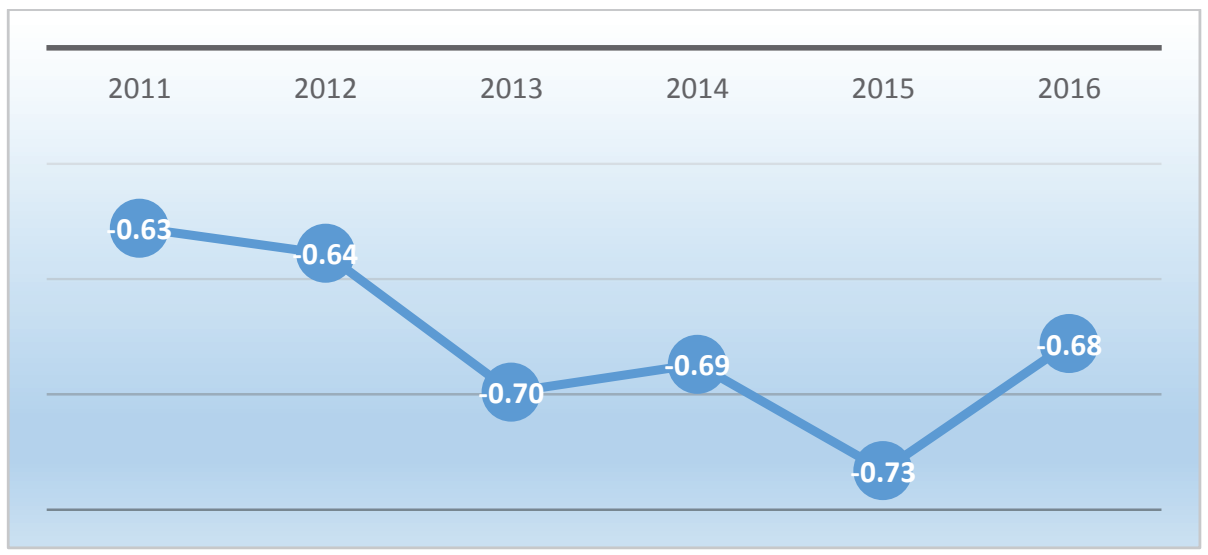

Figura 4. Evolución del Índice de Boone del sistema bancario peruano, 2011-2016 Elaboración en base a estimaciones propias 
Lo anterior no muestra resultados claros sobre la evolución de la competencia y la eficiencia, como sí lo muestran los indicadores del Índice de Lerner presentados por el Banco Mundial. En la figura 5 se aprecia que desde 2011 al 2014 el grado de poder de mercado de la banca peruana se ha venido incrementado, muy por encima de los niveles promedio de los sistemas de Sudamérica e incluso que en mercados grandes como el de Estados Unidos y China.tab

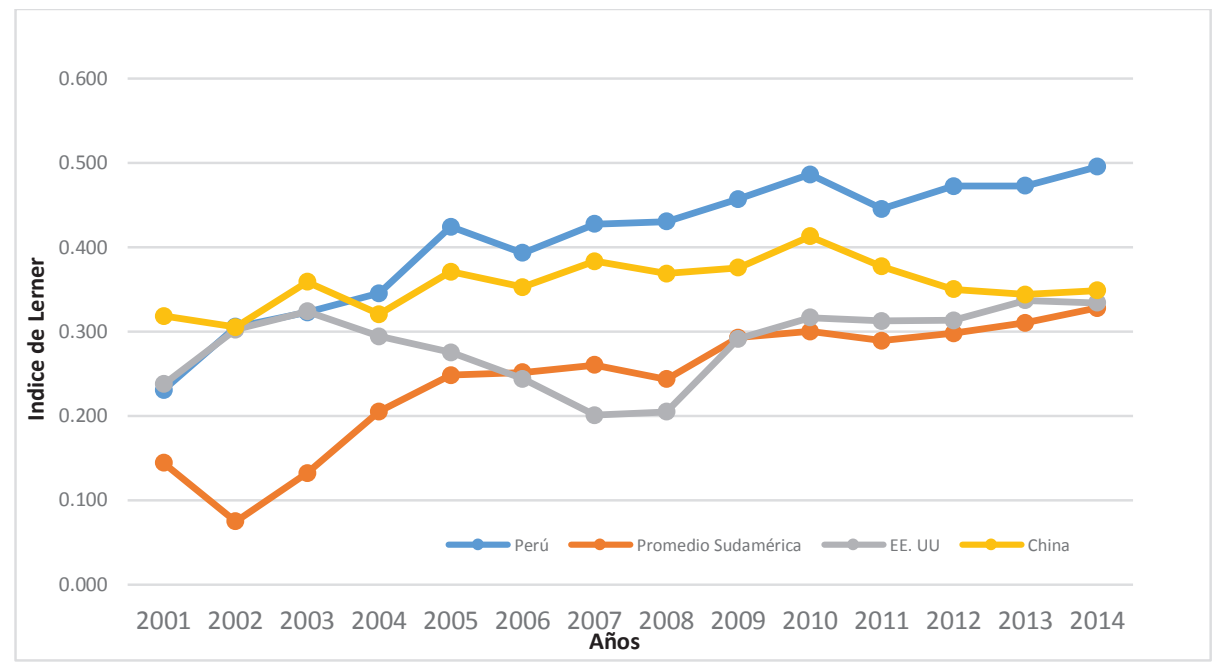

Figura 5. Evolución del índice de Lerner para países seleccionados (2001- 2014) Fuente: Banco Mundial. Elaboración propia

En cuanto a la evolución del Índice por tipos de carteras, en la figura 6, se observa que las carteras, mayorista e hipotecario, si bien presentan poca competencia, muestran una tendencia hacia aumentar su nivel de competencia. Las carteras de crédito minorista y de consumo presentan más competencia confirmando los resultados encontrados para el periodo en su conjunto. Sin embargo, su evolución ha ido en sentidos opuestos ya que la competencia del sector minorista ha incrementado en tanto que la del segmento consumo se ha reducido ligeramente. 


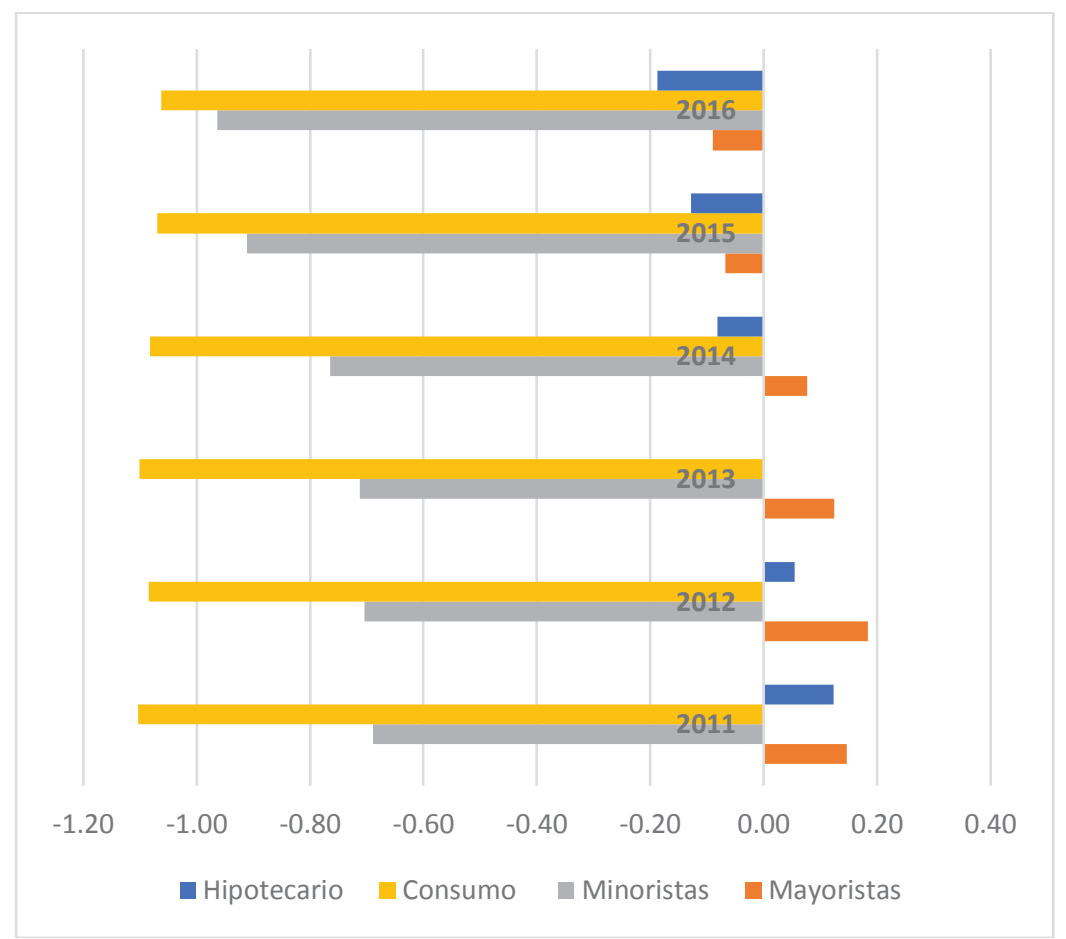

Figura 6. Evolución del Índice de Boone por Segmentos, 2011-2016

Elaboración propia en base a estimaciones

\section{El Î́ndice de Boone versus Î́ndice de Herfindal-Hirschman (IHH)}

Hemos visto que existen dos hipótesis bien definidas sobre la relación entre competencia y concentración, aquí intentaremos relacionar ambas variables a fin de conocer cuál es la relación predominante en base a los resultados encontrados.

Si nos centramos en la evolución de la concentración de las colocaciones del sistema bancario peruano medido por el índice IHH, Figura 7, se observa que la cartera menos concentrada es la de créditos de consumo, las tres carteas restantes presentan un fuerte nivel de concentración, el incremento más marcado se muestra en la cartera de créditos minoristas que desde el 2015 se ha incrementado consistentemente, en línea con la fusión de Mibanco y Edyficar a partir de su compra por el grupo Credicorp. 


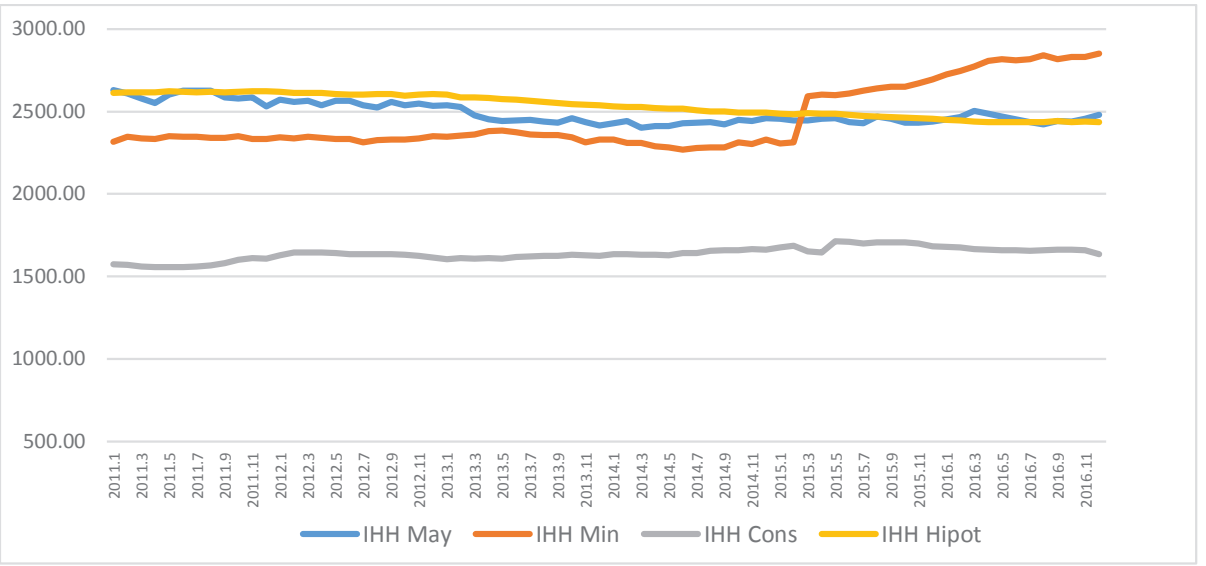

Figura 7. Evolución del Índice IHH de colocaciones del sistema bancario peruano por tipo de Créditos (2011.1-2016.12)

Fuente: SBS. Elaboración propia

Si relacionamos el nivel de concentración de las colocaciones con el nivel de competencia estimado, vimos que los segmentos crediticios que presentan mayor competencia fueron el de crédito minorista y el de consumo, aquí podemos ver que en el sector minorista la mayor concentración si ha impactado directamente en el incremento de la competencia, en tanto, que no sucede lo mismo para el segmento de consumo, el cual es el segmento con menos concentración de colocaciones pero el que tiene, relativamente, un mayor nivel de competencia. Por otra parte, sectores con altos niveles de concentración, pero con ligera tendencia a la baja, como el crédito mayorista y el crédito hipotecario, tienen una ligera tendencia a incrementar la competencia.

Por último, se deja en claro que al no tener estudios concluyentes el estudio de la relación de la competencia y eficiencia del sistema financiero debe ser ampliada a fin de contrastar resultados, nuevos enfoques como el de estimación de eficiencia mediante fronteras estocásticas (análisis envolvente de datos) que den luces sobre la eficiencia técnica y asignativa se hace necesario. Por otra parte, Flores y Watts (2012) aplican el análisis dinámico mediante un modelo de corrección de errores (MCE) en la estimación de conducta de Oligopolio a corto y largo plazo para Chile a fin de contrastar si los niveles de poder de mercado de corto plazo se 
han subestimado (o sobrestimado) en relación a los niveles de largo plazo; y finalmente, tomando como referencia el estudio de Jopen (2013) y la evolución del índice de Lerner a nivel agregado mostrado en la figura 5, se debería estimar dicho índice, no a nivel de tamaño de bancos, sino a niveles de segmentos de mercado como el trabajo acá realizado a fin de contrastar resultados.

\section{Conclusiones}

En el análisis de los costos marginales por tipos de bancos, los Multibancos presentan costos por debajo del promedio del sistema, entre estos, los más eficientes son el Scotiabank y el banco Continental. El banco de Crédito del Perú que representa la tercera parte del sistema, se ubica quinto, por detrás del Banco Santander y el Banco Internacional del Perú. Al parecer, el carácter de bancos internacionales estaría favoreciendo la eficiencia de los bancos. Los Multibancos pequeños presentan costos marginales similares al promedio del sistema. La banca especializada tiene costos por encima del promedio del sistema, siendo los de peor desempeño el banco Azteca y el banco Cencosud, aunque este último con tendencia a decrecer en sus costos marginales.

De acuerdo al índice de Boone, el nivel de competencia del sistema bancario peruano para el periodo 2011-2016, a nivel agregado, es bajo, sin embargo, se habría incrementado ligeramente hasta 2015 para luego decrecer en el 2016. Si observamos el indicador por carteras, los créditos mayoristas y los créditos hipotecarios presentan bajos niveles de competencia, $\mathrm{y}$, al igual que el indicador agregado han tenido un ligero incremento entre 2011 y 2016. La cartera de créditos minoristas y la de créditos de consumo presentan mayores niveles de competencia, sin embargo, estos últimos presentan tendencia a reducirse. Al igual que el trabajo de Alarcón y Olmazabal (2014) para Chile, se encuentran resultados disímiles en distintos segmentos de mercado.

La hipótesis de que la concentración aumenta la competencia y con ello la eficiencia no muestra resultados del todo claros, ya que si bien la cartera de créditos minoristas es altamente concentrada presenta mejores niveles relativos de competencia, no sucede lo mismo con las carteras de créditos mayoristas ni hipotecarios que también son altamente concentradas. Por el 
contrario, la cartera de créditos de consumo siendo la menos concentrada presenta mayor competencia que las demás carteras.

\section{Referencias Bibliográficas}

Aguilar, G. (2016). Competencia en el mercado de microcréditos peruano, una medición utilizando el indicador de Boone. Documento de trabajo ${ }^{\circ}$ 417. Capítulo de Tesis para obtener el grado Doctor en Economía. Pontificia Universidad Católica del Perú. 51p.

Alarcón, C. y Ormazabal, F. (2013). Competencia en el mercado bancario chileno: ¿qué nos dice el indicador Boone? Serie Técnica de Estudios - $\mathrm{N}^{\circ} 014$. Chile: Superintendencia de Bancos e Instituciones Financieras (SBIF). http:// www.asfae.cl/journalmbr/images/stories/pdf/Paper\%201\%20mbr\%20 vol1\%20n6.pdf

Beck, T. (2006). Creating an Efficient Financial System Challenges in a Global Economy. World Bank Policy Research Working Paper 3856. Banco Mundial. Obtenido en http://documents.worldbank.org/curated/ en/642011468134389278/pdf/wps3856.pd

Boone, J. (2004). A New Way to Measure Competition. En (CentER Discussion Paper; Vol. 2004-31. Tilburg: Macroeconomics. Obtenido de https://pure.uvt. nl/portal/files/600949/31.pdf

Boone, J. (2008). Competition, A New Way to Measure. The Economic Journal, p. 1245-1261. Obtenido de http://pareto.uab.cat/xmg/Docencia/IO-en/IOReadings/MeasureCompetition/Boone.pdf

Caminero, S. y Mancebo, E. (2016). Competencia del Sector Bancario Dominicano (2008 - 2016): Una Aproximación por Medio del Indicador Boone. Santo Domingo: Pontificia Universidad Católica Madre y Maestra. Obtenido de http:// investigare.pucmm.edu.do:8080/xmlui/handle/20.500.12060/1848. República Dominicana 108p.

Céspedes, N. y Orrego, F. (2014). Competencia de intermediarios financieros en Perú. Banco Central de Reserva del Perú. Obtenido de http://www.bcrp. gob.pe/docs/Publicaciones/Documentos-de-Trabajo/2014/documento-detrabajo-10-2014.pdf

Claessens, S. y Laeven, L. (2002). Financial Development, Property Rights, and Growth. World Bank Policy Research Working Paper No. 2924. Obtenido de htps://ssrn.com/abstract $=636286$ 
Claessens, S. y Laeven, L. (2004). Competition in the Financial Sector and Growth: A Cross-Country Perspective. En Goodhart C.A.E. (eds), Financial Development and Economic Growth 44p. British Association for the Advancement of Science. Palgrave Macmillan, L. doi:https://doi.org/10.1057/9780230374270_3

Cortez, G. (2006). Competencia y eficiencia en el sector bancario en el Perú 19902005. Pensamiento Crítico. Vol. 6, p. 97-112. Obtenido de https://revistasinvestigacion.unmsm.edu.pe/index.php/econo/article/view/9041/7875

Cortez, G. (2008). Competencia Monopolística y Márgenes Bancarios en el Perú: 1995-2005. Pensamiento Crítico. Vol. 8, p. 115-130. Obtenido de http://revistasinvestigacion.unmsm.edu.pe/index.php/econo/article/view/9155/7984

Croce, H; Macedo, L. y Triunfo, P. (2000). Función de costos: un estudio para los bancos comerciales uruguayo. Universidad de la República. Uruguay. Obtenido de Facultad de Ciencias Sociales. Departamento de Economía. Documento de trabajo Nro. 03/00: http://cienciassociales.edu.uy/departamentodeeconomia/wp-content/uploads/sites/2/2013/archivos/Doc0300.pdf

Del Pozo, C. (2008). Nivel de Competencia en las Microfinanzas, evidencia para el Perú. Obtenido de http://www.bcrp.gob.pe/docs/Proyeccion-Institucional/ Encuentro-de-Economistas/XXVI-EE-2008/XXVI-EE-2008-S15-Paper-DelPozo.pdf

Demirgüç-Kunt; Laeven, L. y Levine, R. (2004). The impact of bank regulations, concentration, and institutions on bank margins, Journal of Money Credit and Banking, Vol. 36. Nro. 2. June. En https://papers.ssrn.com/sol3/papers. cfm?abstract_id $=636392$

Flores, Y. y Watts, D. (2012). Competencia en el sector bancario chileno: una aproximación dinámica. El Trimestre Económico, Vol. LXXIX Nro. 316, p. 865-903. Obtenido de http://www.eltrimestreeconomico.com.mx/index.php/te/article/download/79/79

Florian, León (2014). Measuring competition in banking: A critical review of methods. Serie: Etudes et documents du CERDI. Francia: Clermont-Ferrand. Obtenido de https://halshs.archives-ouvertes.fr/halshs-01015794

Freixas, Xavier y Rochet, Jean-Charles (2008). Microeconomics of banking. Second ed. Cambridge: MIT Press. 389p.

Jiménez, W. (2020). Competencia y eficiencia bancaria en el Perú 2011-2016. Una aproximación mediante el índice de Panzar y Rosse y el Índice de Boone. Tesis para optar el grado de maestro en Economía en la Facultad de Ciencias Económicas de la Universidad Nacional Mayor de San Marcos. Lima - Perú. 124p. 
Jopen, G. (2013) Poder de mercado, intermediación financiera y banca: un enfoque de organización industrial. Economía, Vol. XXXV Nro. 71, p. 75-106. Pontificia Universidad Católica del Perú.

Leuvensteijn, M. (2008). The Boone indicator: identifiying different regimes of competition. The Netherlands: Utrecht School of Economics. Obtenido de http:// dspace.library.uu.nl/bitstream/handle/1874/296438/08-37_1.pdf

Leuvensteijn, M.; Bikker, J; Rixtel, A. y Sorensen, C. (2007) A new approach to measuring competition in the loan markets of the euro area. Germany: European Central Bank. Obtenido de https://www.ecb.europa.eu/pub/pdf/ scpwps/ecbwp768.pdf?8b93d1fe7192d542fa69af9f702789f0

Martin, M. (2009). Transparencia y Concentración bancaria en el Perú. (U. ESAN. Perú, Ed.) México: Pearson Educación. 178p.

Mauricio, P. (2016). El Crédito en el Perú entre los más caros del Mundo. Revistas de Gestión en el Tercer Milenio, Rev. de Investigación de la Fac. de Ciencias Administrativas, UNMSM Vol. 19-II, Nro. 8, Lima, p. 33-40. Obtenido de https://revistasinvestigacion.unmsm.edu.pe/index.php/administrativas/ article/view/13786/12219

Mayorca, E. y Aguilar, G. (2016). Competencia y calidad de cartera en el mercado microfinanciero peruano, 2003-2013. Lima: Departamento de Economía - Pontificia Universidad Católica del Perú. Obtenido de http://revistas.pucp. edu.pe/index.php/economia/article/view/16514/16869

Morón, E., Tejada, J. y Villacorta, A. (2010). Competencia y Concentración en el Sistema Financiero en el Perú. Documento de Discusión DD/10/03, Centro de Investigación de la Universidad del Pacífico, Lima - Perú. Obtenido de http://repositorio.up.edu.pe/bitstream/handle/11354/356/DD1003. pdf?sequence $=1 \&$ isAllowed $=y$

Murillo, G. (2011). Aplicación del Indicador Boone en la Medición de la Competencia del Sistema Bancario Venezolano (2000-2010). Tesis para obtener el grado de magíster en Economía aplicada. Universidad Católica Andrés Bello. Obtenido de http://biblioteca2.ucab.edu.ve/anexos/biblioteca/marc/texto/ AAS2287.pdf. Caracas 159p.

Roengpitya, R. (2010). Measuring the Level of Competition in the Loan Market of the Thai Banking Industry Using the Boone Indicator. Bank of Thailand. Obtenido de https://econpapers.repec.org/paper/bthwpaper/2010-01.htm

Superintendencia de Banca y Seguros -SBS. (2016). Evolución del Sistema Financiero. Obtenido de Boletines Estadísticos: http://www.sbs.gob.pe/app/ stats_net/stats/EstadisticaBoletinEstadistico.aspx? $\mathrm{p}=13 \#$ 
Van Rixtel, A. (2008). Un nuevo indicador de competencia en el sector bancario. Boletín Económico, Banco de España. Banco de España. Obtenido de https:// www.bde.es/f/webbde/SES/Secciones/Publicaciones/InformesBoletinesRevistas/BoletinEconomico/08/Ene/Fich/art5.pdf

Xavier, M. (2013). Eficiencia, Economías de Escala y Alcance en el Sistema Bancario Uruguay. Tesis para obtener el grado de maestro en Economía Internacional. Universidad de la República Uruguay-Facultad de Ciencias Sociales. Obtenido de https://www.colibri.udelar.edu.uy/jspui/handle/123456789/7636. Uruguay $99 \mathrm{p}$.

Zuleta, L.; Misas, M. y Jaramillo, L. (2016). La competencia y la eficiencia en la banca colombiana. Primera ed. (A. Fedesarrollo, Ed.) Bogotá, Colombia: La Imprenta Editores SA.

Zurita, J. (2014). Análisis de la concentración y competencia en el sector bancario. Documento de Trabajo, № 14/23. Obtenido de En https://www.bbvaresearch.com/wp-content/uploads/2014/09/WP-concentraci\%C3\%B3n-ycompetencia-sector-bancario.pdf

\section{Notas al final}

1 El presente artículo de investigación es parte de la Tesis de maestría presentada por el autor en la Facultad de Ciencias Económicas de la Universidad Nacional Mayor de San Marcos cuyo título es Competencia y eficiencia bancaria en el Perú 2011-2016. Una aproximación mediante el Índice de Panzar y Rosse y el Índice de Boone. El proyecto se financió con recursos propios y se ejecutó entre junio de 2017 y junio de 2018. Agradezco al Dr. Abraham Llanos Marcos mi asesor, a Miguel Carbajal Carbajal por su apoyo en el tratamiento estadístico y a dos árbitros anónimos por sus valiosos comentarios.

2 Economista, Universidad Nacional Federico Villareal. Egresado de la maestría de Economía con mención en Finanzas de la Universidad Nacional Mayor de San Marcos. Profesor auxiliar en la escuela de Economía de la Universidad Privada del Norte, Lima. Perú. E-mail: william.jimenez@upn.pe. Código ORCID: 0000-0002-7940-8409

3 Ver http://www.sbs.gob.pe/app/stats_net/stats/EstadisticaBoletinEstadistico.aspx?p=13\#

4 Ver Jiménez, William (2018). Tesis que da origen al presente artículo. Allí se detalla una serie de indicadores del sistema bancario peruano, en cuanto a concentración, rentabilidad, exposición al riesgo, por segmentos crediticios inclusive.

5 Los créditos se han divido en los segmentos de gran empresa, microempresa, hipotecario y de consumo.

6 Los costos marginales provienen de las estimaciones de la tabla 4. Los bancos marcados con asterisco han sido excluidos del cálculo del Índice de Boone por presentar costos marginales negativos lo cual imposibilita el computo. Cabe resaltar que estos no son representativos en términos de participación en el segmento respectivo. 